\title{
Current targets: where are we today?
}

\section{Pringle}

Implementation of the National Service Framework (NSF) for coronary heart disease has particular workload implications for primary care. Studies conducted in general practices known to be representative of practices across the country suggest that, in order to meet current targets, the average general practitioner with a 2000 patient list would need to address 444 disease control measures in 210 patients. These relate primarily to smoking cessation and control of blood pressure, cholesterol, and blood sugar. Despite the enormous scale of cardiovascular disease, a considerable degree of recording and activity is going on within primary care in response to the NSF. However, some areas of cardiovascular care appear to need particular improvement. For example, with respect to lipid management, many high risk patients have not had their lipids measured, while many of those on lipid lowering drugs are not achieving current targets.

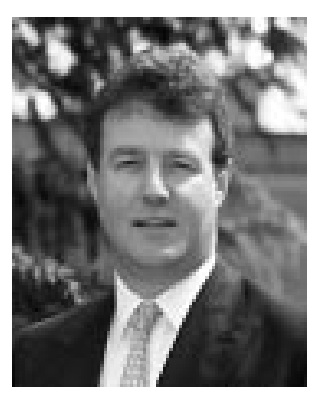

f effective primary and secondary prevention of cardiovascular disease is to be delivered, most of the work will need to be carried out in general practice, with the primary care team taking the prime responsibility for it. While professionals in other sectors will do some of the work, primary care will be the orchestrator of the enterprise.

This paper discusses the general practice workload implications of cardiovascular disease prevention and assesses the progress that has been made to date. It is based on data collected from 17 practices in the Trent region of England, all of which are part of the Trent research practice network. To be included as part of this network, practices must have computer systems compatible with Miquest, a software program for data extraction, and coded data of sufficient accuracy and completeness. It has been shown that patients registered with these practices are representative of the patients throughout Trent in terms of morbidity and sociodemographic characteristics $^{1}$ and Trent is, in general terms, representative of the country as a whole. The data presented are therefore probably generalisable throughout England and possibly further afield in the UK.

Correspondence to: Professor Michael Pringle, School of Community Health Sciences, Division of General Practice, Floor 13 Tower Building, University of Nottingham, NG7 2RD, UK ; mike.pringle@ nottingham.ac.uk

\section{THE SCALE OF THE TASK}

A cross sectional survey ${ }^{2}$ was carried out to investigate the work involved in meeting the targets set out in the National Service Framework (NSF) for coronary heart disease (CHD). ${ }^{3}$ The NSF requires general practitioners (GPs) to identify patients with established CHD or stroke, record their coronary risk factors, and offer appropriate treatment, and to identify and treat patients at high risk of developing CHD.

The scale of the current task can be shown by considering the proportion of patients with the major cardiovascular risk factors. A total of $6 \%$ of the Trent practices' population had diabetes or hypertension but no CHD, while a further $4.5 \%$ had CHD. This equates to 120 and 90 patients, respectively, for a GP with an average list size of 2000. These are high risk patients who should be identified and actively managed. In addition, there will be many other people who have undiagnosed hypertension or diabetes. There are, for example, approximately 700000 people in the UK at present with undiagnosed diabetes.

Data were extracted from practice computers to check whether or not a record had been made of five major risk factors: blood pressure, body mass index, smoking, family history, and cholesterol. All five factors need to be recorded to carry out a full risk assessment. On average, just under one item per patient was missing. The fact that four out of five risk factors were recorded for the average patient shows that GPs are doing quite well, but there is still a large task ahead, particularly since, in addition to the initial recording of those items, considerable work is also required to keep the records up to date with regular recording of blood pressure, weight, cholesterol, and smoking habit.

About $75 \%$ of these high risk patients with hypertension, diabetes or CHD had a blood pressure reading below the previous target level of 160/90 mm Hg. (Data are not available on how many patients had a target systolic pressure-the more important measurement-but raised diastolic pressure.) However, only about a third of patients were reaching the more ambitious target of $<140 / 85 \mathrm{~mm} \mathrm{Hg}$, the currently recommended target blood pressure ${ }^{4}$ (table 1 ). This new target poses a significant challenge; indeed it is arguable whether it is a realistic target at present.

Among patients with diabetes, nearly $50 \%$ had an $\mathrm{HbA}_{\mathrm{lc}}$ measurement of $>7.5 \%$, which is a matter of some concern in terms of glycaemic control. Thirty six per cent of patients with a history of acute myocardial infarction were not being prescribed a $\beta$ blocker, and $28 \%$ of patients with ischaemic heart disease were not recorded as taking aspirin. This last figure could, however, be misleading as many people buy their own aspirin, and over-the-counter use might not be recorded on the practice computer.

In terms of lipid control, $46 \%$ of the study population patients had had their cholesterol recorded. Even if some readings were taken but not recorded on the computer, that figure is not

Abbreviations: CHD, coronary heart disease; GP, general practitioner; NSF, National Service Framework 
Table 1 Patients with coronary heart disease, diabetes or hypertension needing improved disease control measures

\begin{tabular}{ll}
\hline Recording & $\begin{array}{l}\text { Percentage of } \\
\text { patients }\end{array}$ \\
\hline Blood pressure $>160 / 90 \mathrm{~mm} \mathrm{Hg}$ & 26 \\
Blood pressure $>140 / 85 \mathrm{~mm} \mathrm{Hg}$ & 62 \\
Patients with diabetes, $\mathrm{HbA}_{\mathrm{lc}} \geqslant 7.5 \%$ & 48 \\
Patients with history of acute myocardial infarction, & 36 \\
not on $\beta$ blocker & \\
$\begin{array}{l}\text { Patients with ischaemic heart disease, not on aspirin } \\
\text { Cholesterol recorded }\end{array}$ & 28 \\
Of those with fasting serum cholesterol recorded, & 76 \\
cholesterol $>5$ mmol// & 72 \\
Of those with raised fasting serum cholesterol, on & 54 \\
lipid lowering medication & \\
\hline
\end{tabular}

Table 2 Adjusted odds ratio of having a cholesterol of $5 \mathrm{mmol} / \mathrm{l}$ or less ${ }^{5}$

\begin{tabular}{ll}
\hline Drug & Odds ratio \\
\hline Simvastatin & 1.00 \\
Pravastatin & 0.6 \\
Cerivastatin & 0.85 \\
Fluvastatin & 0.45 \\
Atorvastatin & 1.15 \\
Fibrates & 0.24 \\
\hline
\end{tabular}

Table 3 Percentage reduction in cholesterol (pre-prescription to latest) $^{5}$

\begin{tabular}{ll}
\hline Drug & Percentage reduction \\
\hline Simvastatin & 28 \\
Pravastatin & 25 \\
Cerivastatin & 21 \\
Fluvastatin & 24 \\
Atorvastatin & 30 \\
Fibrates & 25 \\
\hline
\end{tabular}

encouraging. The fasting serum cholesterol concentration was $>5 \mathrm{mmol} / \mathrm{l}$ in $72 \%$ of patients in whom it was measured, which is slightly better than might be expected in the general population. Around half the patients with a raised fasting serum cholesterol had been prescribed lipid lowering treatment. The conclusion that can be drawn is that GPs have got considerable work to do to achieve good control of lipid concentrations. This, however, is not a criticism; the data overall show that an enormous amount of activity is under way. It does, however, demonstrate the formidable nature of the task facing primary care practitioners.

To meet the NSF standards, it was calculated that each GP involved in the study would need to address 444 disease control measures, such as giving up smoking, better control of blood pressure, and better control of cholesterol. There were, however, substantial differences between practices in the progress that had been made. Practices varied 14-fold in recording risk factors on computer and four-fold in the need for further disease control measures. Some practices are clearly addressing more of these risk factors than others.

\section{CHOLESTEROL MANAGEMENT}

More recently, data have been collected on cholesterol management in these 17 practices. ${ }^{5}$ The data relate to all
Learning points

- The NSF requires GPs: (1) to identify patients with established CHD or stroke, record their coronary risk factors, and offer appropriate treatment; and (2) to identify and treat patients at high risk of developing CHD. This is an enormous task but we are making visible progress

- There is a need for improvement in lipid managementmany patients have not had their lipids measured, while many of those on lipid lowering drugs are not achieving current targets because statins vary in their effectiveness and dose titration is not being employed sufficiently

patients taking lipid lowering treatment, for whatever clinical indication, and they are therefore not an identical group to those patients in the study discussed above. Of this new group, $65 \%$ had ischaemic heart disease, $12 \%$ had had a stroke, $48 \%$ were hypertensive, and $22 \%$ had diabetes. Others were being treated solely because of their high lipid values.

Of 2469 patients on lipid lowering treatment, 55\% had a last measured cholesterol reading of $\leqslant 5 \mathrm{mmol} / \mathrm{l}$. The data have been broken down to assess lipid control according to the treatment being given. Taking simvastatin as the comparator, it was found that atorvastatin achieved a better outcome and the other drugs all had a notably less successful outcome (table 2). On the basis of these findings, practice protocols that previously recommended fluvastatin as the first choice statin should be reviewed in favour of either simvastatin or atorvastatin.

Table 3 shows the percentage reduction in cholesterol from the pre-treatment recording to the latest recording on treatment. There is not a major difference between simvastatin $(28 \%)$ and atorvastatin $(30 \%)$. The other drugs, including fibrates, do appear to be less effective.

However, perhaps the most important finding from our lipid study is that the results are comparable to those achieved in randomised controlled trials, such as the $4 \mathrm{~S}$ study. ${ }^{6}$ Generally speaking, we would not expect to replicate the achievements of randomised controlled trials in general practice because of the special circumstances under which these trials are conducted-for example, the selective inclusion and exclusion criteria, and long term follow up, plus all the effort that goes into the major studies. This is the first time that British general practice has been shown to achieve the same outcomes in terms of cholesterol reduction as in the major trials.

\section{CONCLUSION}

The scale of the task that primary care is facing in order to meet the NSF standards for prevention of cardiovascular disease in high risk patients is enormous. It is necessary to record the risk factors in order to be able to calculate an individual's risk of CHD, and then to address those risk factors. There is a clear need for improvement in lipid management. Many patients have not had their lipids measured, and many of the patients who are on lipid lowering drugs have not achieved the $5 \mathrm{mmol} / \mathrm{l}$ target. There is a tendency to regard any dose of statin as appropriate without titrating up, if necessary, to the highest recommended dosage.

Given the size of the problem of cardiovascular disease and what primary care teams are being asked to do, the degree of recording and the degree of activity and disease control that is taking place is considerable.

\section{REFERENCES}

1 Hammersley V, Hippisley-Cox J, Wilson A, et al. A comparison of research general practices and their patients with other practices - a cross-sectional study in Trent. Br J Gen Pract 2002;52:463-8. 
2 Hippisley-Cox J, Pringle M. General practice workload implications of the national service framework for coronary heart disease: cross sectional survey. BM 2001;323:269-70.

3 Department of Health. National service framework for coronary heart disease. London: Department of Health, 2000.

4 Ramsay LE, Williams B, Johnston GD, et al, for the British Hypertension Society. Guidelines for management of hypertension: report of the third working party of the British Hypertension Society. J Hum Hypertens 1999; 13:569-92.

5 Hippisley-Cox J, Cater C, Pringle M, et al. A cross-sectional survey of the effectiveness of lipid lowering drugs in lowering serum cholesterol in 17 general practices. BM (in press).

6 Scandinavian Simvastatin Survival Study Group. Randomised trial of cholesterol lowering in $\mathbf{4 4 4 4}$ patients with coronary heart disease: the Scandinavian simvastatin survival study (4S). Lancet 1994;344:1383-9.

\section{DISCUSSION}

Question: You mentioned collecting family history as a risk factor. How do we enter that as a separate factor into our calculations?

Professor Pringle: With the Sheffield tables you adjust the score for a family history but most of the others do not contain this as part of the calculation. However, I think most of us would recognise that knowing about family history enables us to give contextual information to patients, which helps them to understand what we are talking about. We need to try to explain cardiovascular risk to patients in a less technical manner. The more we go into numbers, risk scores, and so on, the less they are likely to fully understand and appreciate what we are saying.

Professor Lewis Ritchie: As far as the Joint British Recommendations are concerned, some authorities recommend $50 \%$ additional weighting for family history - that is, multiply by 1.5 . So, for example, a $10 \%$ risk would increase to $15 \%$ with a positive family history of premature heart disease. I agree that putting the risk into context for the patient is very important.

Question: Are your data from Trent representative of areas with a large ethnic minority population? Also, while we have heard today about considering cardiovascular risk in a multifactorial way, you seemed to be discussing individual risk factors

Professor Pringle: Trent has a fairly representative ethnic mix comparable to the whole of the UK. The study practices are scattered throughout the whole of the area and provide a fairly representative sample. The figures might not hold good for areas with particular needs.
As far as risk calculation is concerned, if GPs' computer systems had risk scores routinely entered then we would begin to use those data. But it is difficult for researchers to attempt to generate a risk score, especially if data are missing, and we have never attempted to do that. It could lead to fallacious results-for example, some of the blood pressure readings may be post-treatment while for most of the risk scores you are meant to use the pre-treatment blood pressure levels. I take your point absolutely, but we were dealing with the best data we had. Of course, we will never achieve 100\% scores on blood pressure control because that would quite clearly involve forcing patients into treatment that they did not want. We have to achieve the best levels that we can without being draconian.

Question: How do we get our political masters to support us in this work? We have successfully achieved over $75 \%$ statin use in secondary prevention and the reward from our primary care trust is that they are going to reduce the community nursing budget and freeze our practice nurse budgets.

Professor Pringle: You are highlighting the extraordinary difficulties at the moment with a cash limited, deprived health service and rising expectations. Primary care is taking the brunt of this. I want to prescribe more statins and, like you, have constraints placed upon me by my PCT. We have got to make sure that the cash is delivered to the health service and to those of us on the front line. With ischaemic heart disease, I think that we have already done wonders with almost no extra resources.

Question: Much of the NSF and the audit selection concentrates on the under $75 \mathrm{~s}$ and the risk factor tables concentrate on the under 75s. Yet those at highest risk are over 75 . Do your data include the over 75 s? We are struggling as it is to collect data for the under 75s. Are we going to collapse under the strain of dealing with the older age group?

Professor Pringle: I have been assured that although the NSF concentrates on the under $75 \mathrm{~s}$, because that is where the data are, we are expected to take older patients into account. The data I reported related to all patients. As far as my practice is concerned, we try not to be ageist about the management of coronary heart disease. However, I think that many of our older patients do take a less interventionist view and want less aggressive management. 